\title{
Additive prognostic value of preoperative plasma glucose concentrations in calves undergoing abdominal surgery
}

\author{
C. K. Lausch, ${ }^{1} \odot$ A. Lorch, ${ }^{1}$ S. Giertzuch, ${ }^{1} \odot$ A. Rieger, ${ }^{1} \odot$ and F. M. Trefz ${ }^{1,2 *} \odot$
}

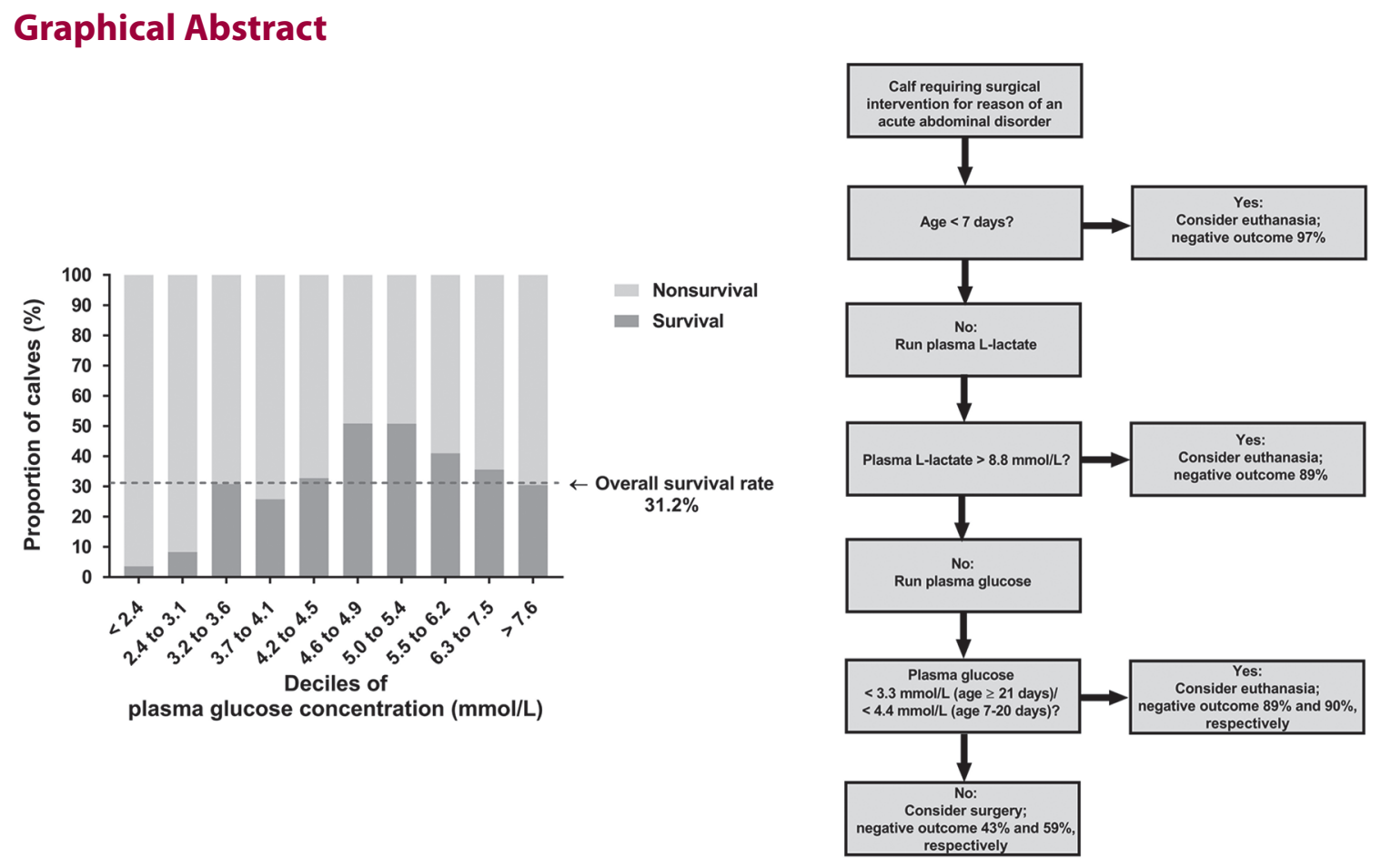

\section{Summary}

Acute abdominal emergencies in calves frequently require surgical intervention and high therapeutic expenditures. The aim of the present study was to assess the prognostic relevance of preoperative plasma glucose concentrations in a retrospective and prospective study population consisting of 586 and 83 affected calves, respectively. We found that hypoglycemia, in addition to hyper-L-lactatemia and low age, was associated with increased mortality risk. Central findings of this study further indicated that hypoglycemia was frequently associated with intraoperative evidence of a septic process within the abdominal cavity.

\section{Highlights}

- Surgical abdominal emergencies in calves are associated with a reserved prognosis.

- Hypoglycemia was indicative of a poor prognosis and serious intraoperative findings such as peritonitis.

- Consideration of plasma glucose improved the accuracy of a prognosis model based on plasma L-lactate and age.

- Survival rates of calves aged $<7 \mathrm{~d}$ and/or with plasma L-lactate $>8.8 \mathrm{mmol} / \mathrm{L}$ were markedly low.

- If plasma L-lactate was $\leq 8.8 \mathrm{mmol} / \mathrm{L}$, surgery was justified when plasma glucose was $\geq 4.4 \mathrm{mmol} / \mathrm{L}$ (age $7-20 \mathrm{~d}$ ) or $\geq 3.3 \mathrm{mmol} / \mathrm{L}$ (age $\geq 21 \mathrm{~d}$ ).

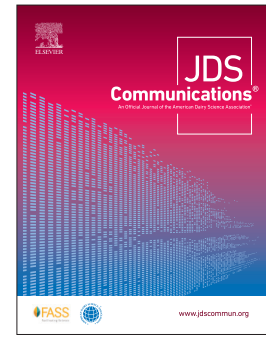

${ }^{1}$ Clinic for Ruminants with Ambulatory and Herd Health Services at the Centre for Clinical Veterinary Medicine, Ludwig-Maximilians-Universität (LMU) Munich, Sonnenstrasse 16, 85764 Oberschleissheim, Germany, ${ }^{2}$ Clinic for Ruminants, Vetsuisse-Faculty, University of Bern, Bremgartenstrasse $109 a, 3012$ Bern, Switzerland. *Corresponding author: florian.trefz@vetsuisse.unibe.ch. @ 2021, The Authors. Published by Elsevier Inc. and Fass Inc. on behalf of the American Dairy Science Association ${ }^{\oplus}$. This is an open access article under the CC BY license (http://creativecommons.org/licenses/by/4.0/). Received June 02, 2021. Accepted August 16, 2021. 


\title{
Additive prognostic value of preoperative plasma glucose concentrations in calves undergoing abdominal surgery
}

\author{
C. K. Lausch, ${ }^{1} \odot$ A. Lorch, ${ }^{1}$ S. Giertzuch, ${ }^{1} \odot$ A. Rieger, ${ }^{1} \odot$ and F. M. Trefz ${ }^{1,2 *} \odot$
}

\begin{abstract}
Surgical abdominal emergencies in calves are associated with a guarded prognosis, especially if neonates are affected. Because hypoglycemia has been associated with sepsis and endotoxemia, this study aimed to assess the prognostic relevance of preoperative plasma glucose concentrations (GLUC) in calves requiring surgery for an acute abdominal disorder. For this purpose, data from retrospective and prospective case series were analyzed, consisting of 586 and 83 hospitalized calves, respectively. The outcomes of calves were evaluated until hospital discharge (both study populations) and for 3 mo following discharge by a phone call to the farmer (prospective study population). For the retrospective study population, the overall survival rate was $31.2 \%$. Calves with a negative outcome (NO) had significantly lower median GLUC $(4.3 \mathrm{mmol} / \mathrm{L})$ than calves with a positive outcome $(\mathrm{PO} ; 5.0 \mathrm{mmol} / \mathrm{L})$. The survival rates of calves with GLUC $<2.4 \mathrm{mmol} / \mathrm{L}$ and 2.4 to $3.1 \mathrm{mmol} / \mathrm{L}$ were 3.6 and $8.3 \%$, respectively. The inclusion of GLUC improved a previous prognostic model based on plasma L-lactate concentration and age. The resulting analyses indicated that NO was associated with low age $(<7 \mathrm{~d})$, hyper-L-lactatemia $(>8.84 \mathrm{mmol} / \mathrm{L}$ ), and GLUC $<4.4 \mathrm{mmol} / \mathrm{L}($ age $7-20 \mathrm{~d})$ and $<3.3 \mathrm{mmol} / \mathrm{L}($ age $\geq 21 \mathrm{~d}$ ), respectively. The area under the receiver operating characteristic curve of this model was 0.79 (95\% confidence interval: $0.76-0.83$ ) and the resulting sensitivity and specificity for $\mathrm{NO}$ at the optimal probability cut-point of 0.69 were 66.7 and $85.8 \%$, respectively. For the prospective study population, the established model had sensitivity and specificity for predicting NO after 3 mo (proportion 24\%) of 61.9 and $85 \%$, respectively. In both study populations, hypoglycemia was significantly associated with intraoperative evidence of a septic process within the abdominal cavity. The present analyses show that hypoglycemia was highly indicative of a poor prognosis and serious intraoperative findings such as peritonitis. Determination of GLUC should therefore be part of the diagnostic work-up in calves suffering from an acute abdominal emergency.
\end{abstract}

A cute abdominal emergencies in calves frequently require surgical intervention and high therapeutic expenditures. These conditions are associated with a grave prognosis, however, as indicated by reported success rates of 24 to $51 \%$ (Iselin et al., 1997; Mulon and Desrochers, 2005; Lausch et al., 2020). This issue has been attributed to irreversible organ damage due to rapid disease progression, concomitant complications such as sepsis and endotoxemia, as well as a high prevalence of peritonitis and malformations if neonatal calves are affected (Iselin et al., 1997; Mulon and Desrochers, 2005; Lausch et al., 2019). Consequently, for economic and animal welfare reasons, there is a need for rapid and easily detectable biomarkers that allow for an objective and accurate prediction of the outcome of therapy in affected animals.

In this context, recent studies (Lausch et al., 2019, 2020; Giertzuch et al., 2020) have focused on the prognostic relevance of plasma L-lactate concentrations (L-LAC), because measurement of this metabolite can be easily performed using handheld L-lactate analyzers such as the Lactate-Pro device (Arkray; Buczinski et al., 2014). These studies revealed that markedly increased L-LAC is associated with an increased mortality risk, but that it is not possible to reliably predict the outcome of affected calves based on a single, preoperative measurement (Lausch et al., 2019, 2020). However, a remarkable finding was that survival rates of affected neonatal calves are low and the predictive accuracy of L-LAC can be improved when the age of the calf is additionally considered (Lausch et al., 2019). Furthermore, hyper-L-lactatemia during the initial postoperative period was shown to be a more reliable prognostic indicator than hyper-L-lactatemia before initiation of therapy (Lausch et al., 2020).

Aside from L-LAC, determination of plasma glucose concentrations (GLUC) might have utility in predicting the outcome of affected calves because hypoglycemia was found to be associated with low survival rates and serious conditions such as peritonitis or clinical evidence of sepsis in critically ill neonatal calves (Trefz et al., 2016, 2017). Hypoglycemia was also observed during experimentally induced sepsis or endotoxemia in calves (Constable et al., 1991a; Kinsbergen et al., 1994a; Ballou et al., 2011), and a recent study reported a negative correlation between plasma endotoxin and GLUC in neonatal diarrheic calves (Gomez et al., 2019). Similar to L-LAC, the availability and validation of glucometers such as the Freestyle Optium Neo H (Abbott) device allow for calf-side measurement of blood glucose concentrations (Karapinar et al., 2020) and could therefore assist in the clinical decision-making process.

The primary aim of the present study was therefore to assess the prognostic utility of preoperative GLUC in study populations

\footnotetext{
${ }^{1}$ Clinic for Ruminants with Ambulatory and Herd Health Services at the Centre for Clinical Veterinary Medicine, Ludwig-Maximilians-Universität (LMU) Munich, Sonnenstrasse 16, 85764 Oberschleissheim, Germany, ${ }^{2}$ Clinic for Ruminants, Vetsuisse-Faculty, University of Bern, Bremgartenstrasse $109 a, 3012$ Bern, Switzerland. *Corresponding author: florian.trefz@vetsuisse.unibe.ch. $\odot 2021$, The Authors. Published by Elsevier Inc. and Fass Inc. on behalf of the American Dairy Science Association ${ }^{\oplus}$. This is an open access article under the CC BY license (http://creativecommons.org/licenses/by/4.0/). Received June 02, 2021. Accepted August 16, 2021.
} 
of hospitalized calves with a broad spectrum of acute abdominal emergencies. A secondary aim was to assess whether consideration of GLUC improves the accuracy of a previously established model based on L-LAC and age (Lausch et al., 2019). Based on the results of previous studies, we hypothesized that low GLUC concentrations in affected animals are associated with a higher mortality risk and that measurement of GLUC provides additional prognostic information.

This study is based on analysis of retrospective and prospective data sets [DS1 and DS2, respectively] that were used in previous studies focusing on the prognostic utility of pre- and postoperative L-LAC measurements in calves with surgical abdominal emergencies (Lausch et al., 2019, 2020). Because of a partial overlap of investigation periods, a subset of calves up to the age of $21 \mathrm{~d}$ in both study populations were part of a much larger data set of 10,060 calves in a previous study on the prevalence, clinical associations, and prognosis of severe hypoglycemia in neonatal calves (Trefz et al., 2016).

Both study populations consisted of calves that required surgery for reasons of an acute abdominal emergency, including cases of abomasal disorders, gastrointestinal ileus, malformations, or peritonitis. For DS1, the medical records of 587 calves (age $\leq 6 \mathrm{mo}$ ) that were admitted to the Clinic for Ruminants with Ambulatory and Herd Health Services, LMU Munich (Oberschleissheim, Germany) between May 2005 and August 2015 were retrospectively analyzed. For DS2, a total of 83 calves (age $\leq 7 \mathrm{mo}$ ) were prospectively enrolled at the same clinic between August 2015 and December 2016. The latter study was approved by the ethics committee of the Centre of Veterinary Clinical Medicine, LMU Munich (permit no. 39-15-01-2015). A decision toward surgical intervention was made on the basis of typical clinical findings (including signs of colic, abdominal distension, tensed abdominal wall, dehydration, scant or absent feces, and a positive right-sided percussion and succussion auscultation) and was supported by an ultrasonographic examination if deemed necessary by the responsible clinician. More details regarding inclusion and exclusion criteria for calves, as well as information about analysis of medical records, clinical examinations, surgical procedures, and treatment of calves have been reported previously (Lausch et al., 2019, 2020). For the present analysis, surgery reports were additionally screened for documented evidence of a septic process within the abdominal cavity, which was defined as necrosis or perforation of an organ of the gastrointestinal tract, signs of fibrinous peritonitis, or severe malodor of the abdominal cavity.

Plasma GLUC concentrations were determined using lithium heparinized blood samples containing potassium fluoride as a glycostatic agent. Samples were collected from the jugular vein on admission to the hospital or in a preoperative situation before any treatment was administered and were analyzed by means of automatic systems [Hitachi 911 (2005-2012) and Cobas c311 (2012-2016); Roche Diagnostics] using the hexokinase method. Hyperglycemia was defined as GLUC as $>6.9 \mathrm{mmol} / \mathrm{L}$ (Kraft and Dürr, 1995). Hypoglycemia was defined as GLUC $<4.4 \mathrm{mmol} / \mathrm{L}$ in neonates aged $<21 \mathrm{~d}$ (Kraft and Dürr, 1995; Trefz et al., 2016) and as $<3.5 \mathrm{mmol} / \mathrm{L}$ in older calves based on findings from a recent study (Yu et al., 2019).

Statistical analyses were performed using SPSS for Windows (version 27.0, IBM Corp.), GraphPad Prism (version 7.01, Graph-
Pad Software), and the partykit (version 1.1-1) package in R (version 3.4.4; https://www.r-project.org/). Values of $P<0.05$ were declared statistically significant. Because most of the data were not normally distributed based on Shapiro-Wilk tests and visual examination of quantile-quantile (QQ) plots, data are presented as medians and interquartile ranges $\left(\mathbf{Q}_{1}-\mathbf{Q}_{3}\right)$. Differences between 5 defined groups of calves based on intraoperative lesion localization were assessed using a Kruskal-Wallis test. For subsequent pairwise comparisons, the Mann-Whitney U-test was used and the level of significance adjusted using the Bonferroni method $(P<0.005)$. In respect to DS1, a positive outcome (PO; survival) was defined as discharge from the hospital, whereas the group of animals with a negative outcome (NO; nonsurvival) consisted of calves that died or were euthanized during hospitalization. For DS2, 2 outcome definitions were used. The outcome of calves was evaluated until hospital discharge and after 3 mo following discharge by means of a phone call to the farmer, as previously described (Lausch et al., 2020).

Mann-Whitney U-tests were used to compare GLUC between calves with PO and NO. Comparisons of categorical parameters between groups were made using a chi-squared test. Also, survival rates in relation to deciles of GLUC were evaluated (Trefz et al., 2017). The predictive ability of GLUC was assessed by means of a receiver operating characteristic (ROC) curve analysis. This included the calculation of the area under the ROC curve (AUCROC) and the associated 95\% confidence interval (95\% CI), as well as the identification of cut-off values that optimized the resulting sensitivity and specificity for prediction of a NO on the basis of the Youden index.

For multivariable modeling of plasma GLUC, L-LAC, and age, DS1 was used as a learning sample and analyzed using classification tree and binary logistic regression analysis, as previously described (Lausch et al., 2019). Two final models were built: the first was based on GLUC and age, and the second additionally included L-LAC data from Lausch et al. (2019) to assess whether GLUC provides prognostic information in addition to L-LAC and age. The predictive abilities of the models were compared by calculating the AUC-ROC and sensitivity, specificity, positive predictive value (PPV), and negative predictive value (NPV) at the optimal cut-point identified using the Youden index. Prospective DS2 was subsequently used as a test sample to assess the predictive utility of the models until hospital discharge and 3 mo later.

For DS1, information on GLUC was not available for 1 calf; data for 586 calves were consequently used in the analysis reported here. The proportion of PO until hospital discharge was $31.2 \%$. Individual diagnoses and respective survival rates are listed in Table 1.

Hypoglycemia was present in 194 calves (33.2\%), whereas hyperglycemia was diagnosed in 76 calves $(13 \%)$. The proportion of hypoglycemic calves was significantly higher $(P<0.001)$ in neonatal calves (age $<21 \mathrm{~d}$; prevalence: $57.9 \%)$ than in older calves (prevalence: $18.9 \%$ ). Calves with a diagnosis of peritonitis had significantly $(P<0.001)$ lower median GLUC (median: 3.7; $\mathrm{Q}_{1}-\mathrm{Q}_{3}$ : $2.6-4.7 \mathrm{mmol} / \mathrm{L} ; \mathrm{n}=148)$ than calves with an abomasal disorder (median: 5.2; $\mathrm{Q}_{1}-\mathrm{Q}_{3}$ : 4.2-6.3 $\mathrm{mmol} / \mathrm{L} ; \mathrm{n}=99$ ) or a problem located in the small intestine (median: $4.6 ; \mathrm{Q}_{1}-\mathrm{Q}_{3}: 3.4-5.7 \mathrm{mmol} / \mathrm{L}$; $\mathrm{n}=131$ ), large intestine (median: $4.5 ; \mathrm{Q}_{1}-\mathrm{Q}_{3}: 3.6-5.5 \mathrm{mmol} / \mathrm{L} ; \mathrm{n}$ $=92$ ), and multiple parts of the gastrointestinal tract (median: 5.5; 
$\mathrm{Q}_{1}-\mathrm{Q}_{3}: 4.4-6.9 \mathrm{mmol} / \mathrm{L} ; \mathrm{n}=116$ ). Review of the surgery reports indicated that $77.8 \%$ of hypoglycemic calves had documented evidence of a septic process within the abdominal cavity compared with $42.2 \%$ calves that presented with normo- or hyperglycemia $(P<0.001)$.

Calves with NO were also younger (median: 3.4; $\mathrm{Q}_{1}-\mathrm{Q}_{3}$ : 1.0-8.6 wk) than calves with a PO (median: $6.0 ; \mathrm{Q}_{1}-\mathrm{Q}_{3}: 4.0-10.0$ wk; $P<0.001$ ) and had lower median GLUC (median: $4.3 \mathrm{Q}_{1}-\mathrm{Q}_{3}$ : 3.1-5.7 mmol/L) than calves with $\mathrm{PO}$ (median: 5.0; $\mathrm{Q}_{1}-\mathrm{Q}_{3}: 4.3-6.0$ $\mathrm{mmol} / \mathrm{L} ; P<0.001)$. The survival rates of calves with GLUC $<2.4$ $\mathrm{mmol} / \mathrm{L}$ (decile 1) and 2.4 to $3.1 \mathrm{mmol} / \mathrm{L}$ (decile 2) were 3.6 and $8.3 \%$, respectively. The AUC-ROC for GLUC as a prognostic indicator was 0.63 (95\% CI: $0.59-0.68 ; P<0.001)$, and an identified cut-point for GLUC $\leq 4.3 \mathrm{mmol} / \mathrm{L}$ had a sensitivity and specificity for the prediction of nonsurvival of 49.9 and $76.5 \%$, respectively.

A classification tree analysis based on age and GLUC (model 1) indicated that nonsurvival was associated with age categories of $<7$ $\mathrm{d}$ and $<25 \mathrm{~d}$, respectively. In calves aged 7 to $24 \mathrm{~d}$, mortality was further associated with GLUC $<4.3 \mathrm{mmol} / \mathrm{L}$ (Figure 1A). A second classification tree analysis on the basis of L-LAC, GLUC, and age (model 2) indicated that nonsurvival was associated with L-LAC $>8.84 \mathrm{mmol} / \mathrm{L}$ and age categories of $<7 \mathrm{~d}$ and $<21 \mathrm{~d}$, respectively. In calves aged 7 to $20 \mathrm{~d}$ and $\geq 21 \mathrm{~d}$, mortality was further associated with GLUC $<4.4 \mathrm{mmol} / \mathrm{L}$ and $<3.3 \mathrm{mmol} / \mathrm{L}$, respectively (Figure
1B). The results of subsequent logistic regression analyses with those categories are shown in Table 2. The AUC-ROC of model 1 (age and GLUC) was 0.72 (95\% CI: $0.68-0.76 ; P<0.001)$ and the resulting sensitivity, specificity, PPV, and NPV for the prediction of nonsurvival at the optimal probability cut-point of 0.70 were $59.1,76.0,84.4$, and $45.7 \%$, respectively. The AUC of model 2 (age, GLUC, and L-LAC) was higher than that for model $1(0.79$; 95\% CI: 0.76-0.83) and the resulting sensitivity, specificity, PPV, and NPV at the optimal cut-point of 0.69 were $66.7,85.8,91.2$, and $54.0 \%$, respectively.

At the individual diagnosis level, a significant difference in GLUC between calves with PO and NO was found only for calves with small intestinal strangulation, torsion of cecum/colon, and mesenteric torsion (Table 1). The AUC-ROC for GLUC in those groups of calves was 0.83 (95\% CI: $0.64-1.0, P=0.049), 0.90$ (95\% CI: $0.74-1.0, P=0.037$ ), and 0.68 (95\% CI: $0.56-0.80, P$ $=0.013)$, respectively. Respective cut-off values for GLUC were $\leq 4.0 \mathrm{mmol} / \mathrm{L}$ in calves with small intestinal strangulation (sensitivity $71.4 \%$, specificity $100 \%$ ) and torsion of cecum/colon (sensitivity $84.6 \%$, specificity $100 \%$ ) and $\geq 6.4 \mathrm{mmol} / \mathrm{L}$ in calves with mesenteric torsion (sensitivity $63.3 \%$, specificity $87.5 \%$ ), respectively.

In DS2, hypoglycemia was present in 25 calves $(30.1 \%)$, whereas hyperglycemia was diagnosed in 15 calves $(18.1 \%)$. The frequency of intraoperative diagnoses was similar to that of

Table 1. Diagnoses, survival rates, age, and plasma glucose concentrations (median and interquartile range, $Q_{1}-Q_{3}$ ) stratified by the outcome of therapy in 586 hospitalized calves with acute abdominal emergencies (retrospective study population)

\begin{tabular}{|c|c|c|c|c|c|c|c|c|}
\hline \multirow[b]{2}{*}{ Diagnosis } & \multirow[b]{2}{*}{$\mathrm{n}$} & \multirow[b]{2}{*}{$\begin{array}{l}\text { Survival } \\
\text { rate (\%) }\end{array}$} & \multirow{2}{*}{$\begin{array}{l}\text { Age }(w k) \\
\text { Median } \\
\left(Q_{1}-Q_{3}\right)\end{array}$} & \multicolumn{2}{|c|}{ Calves with a positive outcome } & \multicolumn{2}{|c|}{ Calves with a negative outcome } & \multirow[b]{2}{*}{$P$-value } \\
\hline & & & & $\mathrm{n}$ & $\begin{array}{c}\text { Glucose }(\mathrm{mmol} / \mathrm{L}) \\
\text { Median }\left(\mathrm{Q}_{1}-\mathrm{Q}_{3}\right)\end{array}$ & $\mathrm{n}$ & $\begin{array}{c}\text { Glucose }(\mathrm{mmol} / \mathrm{L}) \\
\text { Median }\left(\mathrm{Q}_{1}-\mathrm{Q}_{3}\right)\end{array}$ & \\
\hline \multicolumn{9}{|l|}{ Abomasum } \\
\hline Right-sided dilatation & 54 & 72 & $6(4-10)$ & 39 & $5.2(4.6-6.1)$ & 15 & $5.3(4.1-5.8)$ & 0.56 \\
\hline Abomasal volvulus & 39 & 33 & $6(3-10)$ & 13 & $5.6(4.3-7.3)$ & 26 & $4.6(3.3-6.8)$ & 0.24 \\
\hline Miscellaneous $^{1}$ & 6 & 83 & $9(7-13)$ & 5 & $4.9(3.9-6.8)$ & 1 & $8.2\left(\mathrm{NA}^{2}\right)$ & 0.33 \\
\hline \multicolumn{9}{|l|}{ Small intestine } \\
\hline Volvulus & 48 & 44 & $5(1-9)$ & 21 & $4.8(3.9-5.7)$ & 27 & $3.9(2.8-6.7)$ & 0.33 \\
\hline Hernial incarceration & 21 & 67 & $8(2-11)$ & 14 & $4.6(3.6-5.6)$ & 7 & $6.3(3.2-7.8)$ & 0.40 \\
\hline Impaction & 21 & 33 & $5(2-16)$ & 7 & $4.3(3.2-5.9)$ & 14 & $4.6(4.0-5.4)$ & 0.80 \\
\hline Strangulation $^{3}$ & 18 & 22 & $2(2-4)$ & 4 & $5.1(4.4 / 9.9)$ & 14 & $3.4(2.6-4.8)$ & 0.046 \\
\hline Intussusception & 9 & 11 & $2(2-6)$ & 1 & 5.4 (NA) & 8 & $3.5(2.4-5.8)$ & 0.67 \\
\hline Malformations & 8 & 0 & $0(0-1)$ & 0 & NA & 8 & $5.6(4.3-6.7)$ & NA \\
\hline Dislocation & 6 & 50 & $6(4-12)$ & 3 & $5.3(\mathrm{NA})$ & 3 & 3.7 (NA) & 1.0 \\
\hline \multicolumn{9}{|l|}{ Large intestine } \\
\hline Malformations & 39 & 0 & $0(0-1)$ & 0 & NA & 39 & $4.7(3.6-5.8)$ & NA \\
\hline Intussusception & 21 & 24 & $3(2-6)$ & 5 & $5.4(3.5-6.1)$ & 16 & $4.4(4.0-5.0)$ & 0.45 \\
\hline Torsion of cecum/colon & 16 & 19 & $4(2-10)$ & 3 & $4.6(\mathrm{NA})$ & 13 & $3.2(2.6-3.8)$ & 0.037 \\
\hline Cecal dilatation & 10 & 40 & $6(5-15)$ & 4 & $5.4(4.9-6.8)$ & 6 & $5.0(4.3-5.9)$ & 0.48 \\
\hline Impaction & 6 & 50 & $12(5-17)$ & 3 & $6.0(\mathrm{NA})$ & 3 & 4.9 (NA) & 0.70 \\
\hline \multicolumn{9}{|l|}{ Multiple parts } \\
\hline Mesenteric torsion & 73 & 33 & $9(6-11)$ & 24 & $5.1(4.4-6.0)$ & 49 & $6.9(4.3-9.8)$ & 0.013 \\
\hline Bloat/gas colic ${ }^{4}$ & 32 & 84 & $6(4-7)$ & 27 & $5.3(4.6-6.6)$ & 5 & $5.0(3.8-5.8)$ & 0.31 \\
\hline Paralytic ileus & 11 & 46 & $8(2-11)$ & 5 & $5.4(3.3-6.4)$ & 6 & $3.7(2.5-4.2)$ & 0.25 \\
\hline \multicolumn{9}{|l|}{ Abdominal cavity } \\
\hline Peritonitis ${ }^{5}$ & 148 & 3 & $3(1-8)$ & 5 & $3.8(3.6-4.5)$ & 143 & $3.6(2.6-4.8)$ & 0.54 \\
\hline
\end{tabular}

${ }^{1}$ Including 3 calves with abomasal impaction and 3 calves with an incarceration of the abomasum in an umbilical hernia.

${ }^{2}$ Not applicable.

${ }^{3}$ Intestinal strangulations through intraabdominal adhesions, navel structures, or mesenteric defects.

${ }^{4}$ Characterized by gaseous distension of multiple parts of the gastrointestinal tract.

${ }^{5}$ Peritonitis was considered to be related to perforated abomasal ulcers ( $n=39$; of which 30 calves had omental bursitis), intestinal contusion or perforation $(n=35)$, infection of intraabdominal umbilical structures $(n=28)$, intestinal wall necrosis $(n=22)$, septicemia $(n=21)$, rupture of the abomasum $(n=2)$, and wound dehiscence after previous laparotomy $(n=1)$. 

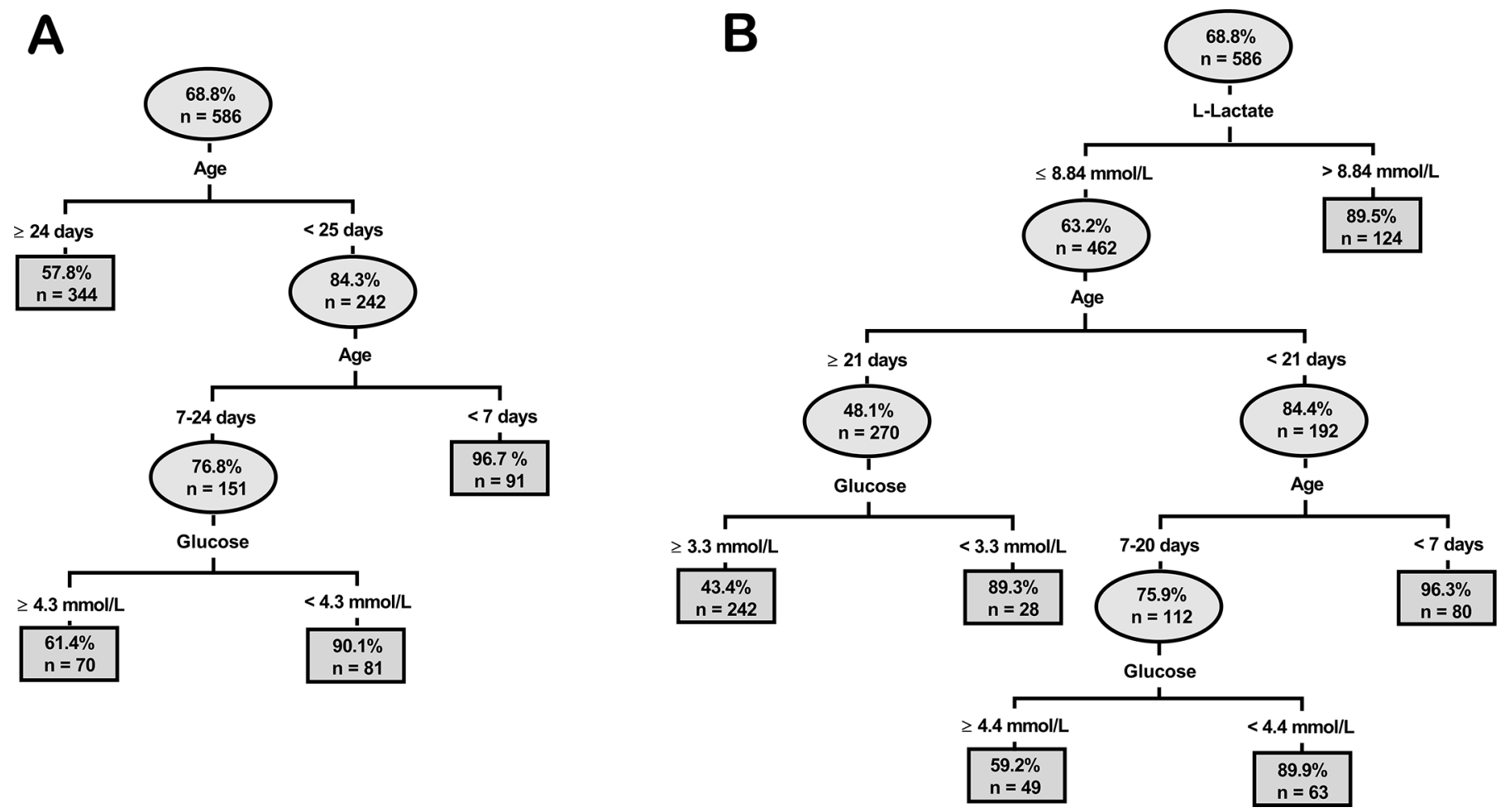

Figure 1. Classification trees illustrating the prognostic value of preoperative plasma glucose concentrations in addition to age (A) and in addition to age and plasma L-lactate concentrations (B) in 586 calves with surgical abdominal emergencies. Ovals and squares represent subsets of the population and indicate the total number of calves in the subset and the respective actual percentages of these calves with a negative outcome. Lines leaving the ovals illustrate the partitioning of a subgroup based on a classification variable and its cut-point value that was predictive of mortality in these animals. Branches to the left indicate subgroups with higher survival rates, whereas branches to the right indicate subgroups with lower survival rates. Figure B modified, with permission, from Lausch et al. (2019).

Table 2. Multivariable logistic regression models ${ }^{1}$ for identifying associations between categories of plasma glucose concentrations and age (model 1) as well as between categories of age and plasma glucose and L-lactate concentrations (model 2) with nonsurvival in 586 calves with acute abdominal emergencies

\begin{tabular}{|c|c|c|c|c|c|}
\hline Variable & Coefficient & SE & Odds ratio (OR) & $95 \% \mathrm{Cl}$ for OR & $P$-value \\
\hline \multicolumn{6}{|l|}{ Model 1} \\
\hline Intercept & 0.027 & 0.125 & & & 0.83 \\
\hline \multicolumn{6}{|l|}{ Age } \\
\hline$<7 d$ & 2.894 & 0.60 & 18.1 & $5.6-58.6$ & $<0.001$ \\
\hline 7 to $24 \mathrm{~d}$ & 0.712 & 0.228 & 2.04 & $1.30-3.19$ & 0.002 \\
\hline$\geq 25 \mathrm{~d}$ & Referent & & & & \\
\hline \multicolumn{6}{|l|}{ Glucose } \\
\hline$<4.3 \mathrm{mmol} / \mathrm{L}$ & 0.974 & 0.210 & 2.65 & $1.76-4.0$ & $<0.001$ \\
\hline$\geq 4.3 \mathrm{mmol} / \mathrm{L}$ & Referent & & & & \\
\hline \multicolumn{6}{|l|}{ Model 2} \\
\hline Intercept & -0.349 & 0.142 & & & 0.014 \\
\hline \multicolumn{6}{|l|}{ Age } \\
\hline$<7 d$ & 3.151 & 0.604 & 23.4 & $7.1-76.4$ & $<0.001$ \\
\hline 7 to $20 \mathrm{~d}$ & 0.927 & 0.259 & 2.53 & $1.52-4.20$ & $<0.001$ \\
\hline$\geq 21 \mathrm{~d}$ & Referent & & & & \\
\hline \multicolumn{6}{|l|}{ L-Lactate } \\
\hline$>8.84 \mathrm{mmol} / \mathrm{L}$ & 1.94 & 0.325 & 6.97 & $3.69-13.16$ & $<0.001$ \\
\hline$\leq 8.84 \mathrm{mmol} / \mathrm{L}$ & Referent & & & & \\
\hline \multicolumn{6}{|l|}{ Glucose } \\
\hline$<3.3$ & 1.857 & 0.381 & 6.40 & $3.03-13.5$ & $<0.001$ \\
\hline 3.3 to $4.3 \mathrm{mmol} / \mathrm{L}$ & 0.495 & 0.244 & 1.64 & $1.02-2.65$ & 0.042 \\
\hline$\geq 4.4 \mathrm{mmol} / \mathrm{L}$ & Referent & & & & \\
\hline
\end{tabular}


DS1 (Lausch et al., 2020). During surgery, 72\% of hypoglycemic calves had evidence of a septic process within the abdominal cavity compared with $34.4 \%$ of calves that presented with normo- or hyperglycemia $(P=0.002)$. The proportion of calves with PO until hospital discharge and after 3 mo were 29 and 24\%, respectively. For model 1 (age and GLUC), the sensitivity, specificity, PPV, and NPV for the prediction of nonsurvival until hospital discharge (and after $3 \mathrm{mo}$ ) at the previously identified probability cut-point of 0.70 were $54.2 \%(52.4 \%), 75 \%(75.0 \%), 84.2 \%(86.8 \%)$, and $40.0 \%$ (33.3\%), respectively. For model 2 (age, GLUC, L-LAC), the values for sensitivity, specificity, PPV, and NPV for the prediction of NO until hospital discharge (and after $3 \mathrm{mo}$ ) at the probability cutpoint of 0.69 were $62.7 \%(61.9 \%), 79.2 \%(85 \%), 88.0 \%(92.9 \%)$, and $46.3 \%(41.5 \%)$, respectively.

Results of this study indicate, therefore, that hypoglycemia is a relatively common finding in calves with surgical abdominal emergencies and associated with fatal intraoperative findings and a markedly increased risk for NO. Furthermore, consideration of GLUC improved the diagnostic accuracy of a previously established model based on L-LAC and age (ROC-AUC: 0.75; Lausch et al., 2019), as indicated by a slightly higher ROC-AUC of 0.79 with model 2. Analysis of the prospective data set largely confirmed the predictive accuracy of the identified factors, which suggests that they are also a good indicator for NO until 3 mo following discharge.

Decreased GLUC is a well-known feature of calves that experience fasting or the feeding of diets that do not meet energy demands (Kinsbergen et al., 1994b; Marcato et al., 2020; Roadknight et al., 2021). Severity and duration of illness and a concomitant alteration of milk intake provide an obvious explanation for the association between hypoglycemia and NO in calves of the present study. Furthermore, it needs to be considered that this and our previous analyses (Lausch et al., 2019, 2020) showed that survival rates of neonatal calves with acute abdominal disorders are markedly low, which is clearly related to the high prevalence of peritonitis and intestinal malformations such as atresia coli in these animals. Moreover, in our experience, strangulating disorders are characterized by a more rapid disease progression in young calves than in older calves or adult cattle. As in a previous study (Trefz et al., 2016), the reported association between hypoglycemia and outcome of therapy might therefore have been confounded by age, because hypoglycemia was more prevalent in younger calves and low age was associated with a markedly increased mortality risk. This issue was addressed, however, by the classification tree analyses, which indicated that determination of preoperative GLUC provides prognostic information in addition to age.

Analysis of intraoperative findings revealed an association between hypoglycemia and intraoperative evidence of a septic process within the abdominal cavity. This finding is in line with experimental studies in pigs and rats, where a hypoglycemic response was observed after induction of peritonitis and sepsis by means of cecal puncture and ligation or intraperitoneal administration of an Escherichia coli culture (Kazarian et al., 1994; Liao et al., 2013; Maitra et al., 2000). Previous studies further reported an association between hypoglycemia and endotoxemia, sepsis, and systemic inflammatory response syndrome in critically ill calves (Lofstedt et al., 1999; Trefz et al., 2016; Gomez et al., 2019). Generalized peritonitis was also found in $37 \%$ of severely hypoglycemic hos- pitalized neonatal calves (Trefz et al., 2016), and investigations in adult cattle have shown that septic peritonitis is associated with decreased glucose concentrations in peritoneal fluid and an increased blood-to-peritoneal fluid glucose difference (Wittek et al., 2010). However, peritonitis in the latter study was not reported to be associated with decreased GLUC, which might be related to higher energy reserves and differences in glucose metabolism in adult cattle compared with suckling calves, which compromised the vast majority of the present study populations. This assumption is also supported by observations that acute abdominal disorders in adult cattle are frequently associated with hyperglycemia (Constable et al., 1991b; Anderson et al., 1993; Dennison et al., 2002).

At the individual diagnosis level, GLUC was only associated with $\mathrm{NO}$ in calves with small intestinal strangulation, torsion of cecum/colon, and mesenteric torsion. Interestingly, NO in calves with mesenteric torsion was associated with increased GLUC. This condition is characterized by an acute to peracute course with clinical signs of fulminant colic, abdominal pain, and frequently cardiovascular shock (Anderson et al., 1993; Rademacher et al., 1995; Desrochers and Anderson, 2016). Calves are predisposed to this condition during the weaning period when they still consume considerable amounts of milk or milk replacer in addition to solid feed (Rademacher et al., 1995). The association between increased GLUC and NO in these calves was therefore likely related to a higher age category (Table 1) and metabolic differences compared with younger calves, but also probably by a higher degree of stress, abdominal pain, and dehydration compared with calves with other disease conditions.

Detection of GLUC alterations in critically ill calves requires measurement of blood glucose concentrations. This can be easily performed by means of portable point-of-care glucometers that allow for calf-side measurement of GLUC under ambulatory farm settings. However, it needs to be considered that currently available glucometers are optimized for human use, which was reported to result in limited accuracies when applied to cattle (Megahed et al., 2015; Karapinar et al., 2020). Despite these shortcomings, a recent study in critically ill neonatal calves reported a sensitivity and specificity for detecting GLUC $\leq 3 \mathrm{mmol} / \mathrm{L}$ using a human glucometer of 92.7 and $97.4 \%$, respectively (Karapinar et al., 2020). These findings suggest that currently available glucometers provide test results that are sufficiently reliable for clinical purposes in calves, which markedly facilitates the applicability of our findings in the field.

Although this study provided additional information with respect to prognostic factors in calves with acute abdominal emergencies, it has some limitations that were also considered relevant in our previous analyses (Lausch et al., 2019, 2020). This included the preselection of cases that are referred to university teaching hospitals, the potential impact of euthanasia on the outcome, and interindividual treatment variations that might be especially relevant for calves in the retrospective DS1. Furthermore, our analyses are based on a single measurement of GLUC upon admission or in a preoperative situation. Therefore, it is likely that this single measurement represents a "snapshot value" that could have been influenced by external factors, including stress or any pretreatment activities.

In conclusion, the present analyses demonstrate that marked hypoglycemia in calves with surgical abdominal emergencies is 
associated with low survival rates and should alert the examiner to the presence of a septic process within the abdominal cavity. Our findings indicate that euthanasia should be considered in affected calves $<7 \mathrm{~d}$ of age or with $\mathrm{L}-\mathrm{LAC}>8.84 \mathrm{mmol} / \mathrm{L}$, or both, based on observed mortality rates of 96.7 and $89.5 \%$, respectively. In calves with $\mathrm{L}-\mathrm{LAC} \leq 8.84 \mathrm{mmol} / \mathrm{L}$, GLUC of $\geq 4.4 \mathrm{mmol} / \mathrm{L}$ (age $7-20 \mathrm{~d}$ ) and $\geq 3.3 \mathrm{mmol} / \mathrm{L}$ (age $\geq 21 \mathrm{~d}$ ) were identified as clinically useful cut-points for a decision toward a surgical treatment trial. This was supported by observed survival rates of 40.8 and $56.6 \%$ compared with 10.1 and $10.7 \%$ in calves below those cut-point values, respectively.

\section{References}

Anderson, D. E., P. D. Constable, G. St Jean, and B. L. Hull. 1993. Smallintestinal volvulus in cattle: 35 cases (1967-1992). J. Am. Vet. Med. Assoc. 203:1178-1183.

Ballou, M. A., C. J. Cobb, L. E. Hulbert, and J. A. Carroll. 2011. Effects of intravenous Escherichia coli dose on the pathophysiological response of colostrum-fed Jersey calves. Vet. Immunol. Immunopathol. 141:76-83. https://doi.org/10.1016/j.vetimm.2011.02.008.

Buczinski, S., E. Doré, G. Boulay, and D. Francoz. 2014. Validation of the handheld Lactate-Pro analyzer for measurement of blood L-lactate concentration in cattle. Vet. Clin. Pathol. 43:567-572. https://doi.org/10.1111/ vcp. 12185.

Constable, P. D., L. M. Schmall, W. W. Muir, and G. F. Hoffsis. 1991a. Respiratory, renal, hematologic, and serum biochemical effects of hypertonic saline solution in endotoxemic calves. Am. J. Vet. Res. 52:990-998.

Constable, P. D., G. St Jean, B. L. Hull, D. M. Rings, and G. F. Hoffsis. 1991b. Preoperative prognostic indicators in cattle with abomasal volvulus. J. Am. Vet. Med. Assoc. 198:2077-2085.

Dennison, A. C., D. C. VanMetre, R. J. Callan, P. Dinsmore, G. L. Mason, and R. P. Ellis. 2002. Hemorrhagic bowel syndrome in dairy cattle: 22 cases (1997-2000). J. Am. Vet. Med. Assoc. 221:686-689. https://doi.org/10 .2460/javma.2002.221.686.

Desrochers, A., and D. E. Anderson. 2016. Intestinal Surgery. Vet. Clin. North Am. Food Anim. Pract. 32:645-671. https://doi.org/10.1016/j.cvfa.2016 .05 .007 .

Giertzuch, S., A. Lorch, C. K. Lausch, G. Knubben-Schweizer, and F. M. Trefz. 2020. Prognostic utility of pre- and postoperative plasma 1-lactate measurements in hospitalized cows with acute abdominal emergencies. J. Dairy Sci. 103:11769-11781. https://doi.org/10.3168/jds.2020-19102.

Gomez, D. E., J. C. Rodriguez-Lecompte, J. Lofstedt, L. G. Arroyo, R. Nino-Fong, and J. T. McClure. 2019. Detection of endotoxin in plasma of hospitalized diarrheic calves. J. Vet. Emerg. Crit. Care (San Antonio) 29:166-172. https://doi.org/10.1111/vec.12815.

Iselin, U., J. Lischer, H. Stocker, and A. L. Steiner. 1997. Kolik beim Kalb, eine retrospektive Studie über 40 Fälle. Wien. Tierärztl. Mschrift. 84:20-25.

Karapinar, T., K. C. Tumer, and S. Buczinski. 2020. Evaluation of the Freestyle Optium Neo H point-of-care device for measuring blood glucose concentrations in sick calves. J. Vet. Intern. Med. 34:1650-1656. https://doi.org/ 10.1111/jvim. 15794.

Kazarian, K. K., P. W. Perdue, W. Lynch, A. Dziki, J. Nevola, C.-H. Lee, I. Hayward, T. Williams, and W. R. Law. 1994. Porcine peritoneal sepsis: Modeling for clinical relevance. Shock 1:201-212. https://doi.org/10.1097/ 00024382-199403000-00008.

Kinsbergen, M., R. M. Bruckmaier, and J. W. Blum. 1994a. Metabolic, endocrine and haematological responses to intravenous E. coli endotoxin administration in 1-week-old calves. Zentralbl Veterinarmed A 41:530-547. https://doi.org/10.1111/j.1439-0442.1994.tb00121.x.

Kinsbergen, M., H. P. Sallmann, and J. W. Blum. 1994b. Metabolic, endocrine and haematological changes in 1-week-old calves after milk intake, in re- sponse to fasting and during total parenteral nutrition. Zentralbl Veterinarmed A 41:268-282. https://doi.org/10.1111/j.1439-0442.1994.tb00094.x.

Kraft, W., and U. Dürr. 1995. Klinische Labordiagnostik in der Tiermedizin. 3rd ed. Schattauer Verlagsgesellschaft.

Lausch, C. K., A. Lorch, S. Giertzuch, A. Rieger, G. Knubben-Schweizer, and F. M. Trefz. 2020. Prognostic relevance of pre- and postoperative plasma L-lactate measurements in calves with acute abdominal emergencies. J. Dairy Sci. 103:1856-1865. https://doi.org/10.3168/jds.2019-17224.

Lausch, C. K., A. Lorch, G. Knubben-Schweizer, A. Rieger, and F. M. Trefz. 2019. Prognostic value of preoperative plasma L-lactate concentrations in calves with acute abdominal emergencies. J. Dairy Sci. 102:10202-10212. https://doi.org/10.3168/jds.2019-16871.

Liao, M.-H., S.-J. Chen, C.-M. Tsao, C.-C. Shih, and C.-C. Wu. 2013. Possible biomarkers of early mortality in peritonitis-induced sepsis rats. J. Surg. Res. 183:362-370. https://doi.org/10.1016/j.jss.2013.01.022.

Lofstedt, J., I. R. Dohoo, and G. Duizer. 1999. Model to predict septicemia in diarrheic calves. J. Vet. Intern. Med. 13:81-88. https://doi.org/10.1111/j .1939-1676.1999.tb01134.x.

Maitra, S. R., M. M. Wojnar, and C. H. Lang. 2000. Alterations in tissue glucose uptake during the hyperglycemic and hypoglycemic phases of sepsis. Shock 13:379-385. https://doi.org/10.1097/00024382-200005000-00006.

Marcato, F., H. van den Brand, B. Kemp, B. Engel, M. Wolthuis-Fillerup, and K. van Reenen. 2020. Effects of pretransport diet, transport duration, and type of vehicle on physiological status of young veal calves. J. Dairy Sci. 103:3505-3520. https://doi.org/10.3168/jds.2019-17445.

Megahed, A. A., M. W. H. Hiew, J. R. Townsend, J. B. Messick, and P. D. Constable. 2015. Evaluation of an electrochemical point-of-care meter for measuring glucose concentration in blood from periparturient dairy cattle. J. Vet. Intern. Med. 29:1718-1727. https://doi.org/10.1111/jvim.13608.

Mulon, P.-Y., and A. Desrochers. 2005. Surgical abdomen of the calf. Vet. Clin. North Am. Food Anim. Pract. 21:101-132. https://doi.org/10.1016/j.cvfa .2004.12.004.

Rademacher, G., G. Dirksen, and W. Klee. 1995. Diagnose, Therapie und Prognose der Darmscheibendrehung beim Rind. Tierarztl. Umsch. 50:271-280.

Roadknight, N., P. Mansell, E. Jongman, N. Courtman, D. McGill, G. Hepworth, and A. Fisher. 2021. Blood parameters of young calves at abattoirs are related to distance transported and farm of origin. J. Dairy Sci. 104:9164-9172. https://doi.org/10.3168/jds.2020-19729.

Trefz, F. M., M. Feist, and I. Lorenz. 2016. Hypoglycaemia in hospitalised neonatal calves: Prevalence, associated conditions and impact on prognosis Vet. J. 217:103-108. https://doi.org/10.1016/j.tvj1.2016.10.001.

Trefz, F. M., I. Lorenz, A. Lorch, and P. D. Constable. 2017. Clinical signs, profound acidemia, hypoglycemia, and hypernatremia are predictive of mortality in 1,400 critically ill neonatal calves with diarrhea. PLoS One 12:e0182938. https://doi.org/10.1371/journal.pone.0182938.

Wittek, T., A. Grosche, L. F. Locher, and M. Fürll. 2010. Diagnostic accuracy of d-dimer and other peritoneal fluid analysis measurements in dairy cows with peritonitis. J. Vet. Intern. Med. 24:1211-1217. https://doi.org/10.1111/ j.1939-1676.2010.0548.x.

Yu, K., F. Canalias, D. Solà-Oriol, L. Arroyo, R. Pato, Y. Saco, M. Terré, and A. Bassols. 2019. Age-related serum biochemical reference intervals established for unweaned calves and piglets in the post-weaning period. Front Vet. Sci. 6:123. https://doi.org/10.3389/fvets.2019.00123.

\section{Notes}

C. K. Lausch (1) https://orcid.org/0000-0001-5350-4702

S. Giertzuch (1) https://orcid.org/0000-0002-0677-8855

A. Rieger (1) https://orcid.org/0000-0002-6826-4497

F. M. Trefz 이 https://orcid.org/0000-0002-9784-8889

There was no external funding for this study.

The authors have not stated any conflicts of interest. 